\title{
Vessel Wall Enhancement in Treated Unruptured Aneurysms
}

W e read with great interest the article "Vessel Wall Enhancement in Unruptured Intracranial Aneurysms: An Indicator for Higher Risk of Rupture? High-Resolution MR Imaging and Correlated Histologic Findings," published in the American Journal of Neuroradiology by Larsen et al. ${ }^{1}$ The authors found that wall enhancement in vessel wall MR imaging is associated with inflammatory cell invasion, neovascularization, and the presence of vasa vasorum, factors that provide valuable information for unruptured aneurysm risk stratification. ${ }^{1}$ Previous studies have suggested that aneurysm wall inflammation may precede, rather than result from, rupture. In as much as inflammation affects aneurysm growth and rupture, vessel wall enhancement may be a marker of rupture risk. $^{2}$

However, we would like to discuss the clinical importance of the presence of aneurysm wall enhancement, detected by highresolution MR imaging after treatment. We present the case of a 68-year-old woman with an unruptured anterior cerebral artery aneurysm treated with a flow diverter. Follow-up 3T MR angiography 1 month after flow-diverter placement showed magnetic susceptibility artifacts in the Al portion of the right anterior cerebral artery, secondary to the flow diverter. The aneurysm, which measured $1.7 \times 1.5 \mathrm{~cm}$, had a T1-hyperintense signal within it due to thrombosis or slow flow induced by the treatment. Subtraction of the precontrast from the postcontrast enhanced vessel wall images revealed a thin rim of enhancement around the aneurysm wall, without edema in the surrounding parenchyma (Figure). The patient was asymptomatic at the time of the examination and is being monitored as an outpatient.

A thin rim of circumferential contrast enhancement around a treated aneurysm is commonly seen in contrast-enhanced TOF-MR angiography following treatment and should be an expected finding. This contrast enhancement is thought to be attributed to a combination of organized peripherally distributed intra-aneurysmal thrombosis, vasa vasorum within the adventitial layer of the aneurysm wall, and/or growth of vascularized tissue into an implanted coil mass or flow diverter due to inflammation or healing. ${ }^{3}$

Guan et $\mathrm{al}^{4}$ described 3 patients with intracranial aneurysms treated with flow diversion. Post treatment vessel wall high-resolution MR imaging to evaluate aneurysm obliteration revealed intra-aneurysm thrombus development, reduced aneurysm filling, and vessel wall enhancement of the aneurysm sac in all 3 patients. Although the authors did not discuss the significance of the wall enhancement detected by vessel wall imaging, all 3 patients were clinically well and in outpatient follow-up, like the case presented here. In such post treatment scenarios, vessel wall enhancement detected by high-resolution vessel wall MR imaging may be of no clinical significance and not a sign of elevated rupture risk, though this technique is more sensitive to inflammatory changes, atherosclerotic plaques, arterial dissection, and other causes of intracranial arterial narrowing than TOF-MR angiography. ${ }^{2}$

Hence, whereas vessel wall enhancement of nontreated intracranial aneurysms seen in high-resolution vessel wall imaging may be a marker of rupture risk, ${ }^{1,2}$ wall enhancement of a treated aneurysm may not necessarily be pathologic, though there is a lack of studies addressing the utility of vessel wall imaging in treated aneurysms in the literature.

\section{REFERENCES}

1. Larsen $\mathrm{N}$, von der Brelie $\mathrm{C}$, Trick $\mathrm{D}$, et al. Vessel wall enhancement in unruptured intracranial aneurysms: an indicator for higher risk of rupture? High-resolution MR imaging and correlated histologic findings. AJNR Am J Neuroradiol 2018;39:1617-21 CrossRef Medline

2. Mandell DM, Mossa-Basha M, Qiao Y, et al; Vessel Wall Imaging Study Group of the American Society of Neuroradiology. Intracranial vessel wall MRI: principles and expert consensus recommendations of the American Society of Neuroradiology. AJNR Am J Neuroradiol 2017; 38:218-29 CrossRef Medline

3. Wallace RC, Karis JP, Partovi S, et al. Noninvasive imaging of treated cerebral aneurysms, Part I: MR angiographic follow-up of coiled aneurysms. AJNR Am J Neuroradiol 2007;28:1001-08 CrossRef Medline

4. Guan J, Karsy M, McNally S, et al. High-resolution magnetic resonance imaging of intracranial aneurysms treated by flow diversion. Interdisciplinary Neurosurgery 2017;10:69-74 CrossRef

(1)D.G. Corrêa

(1) R.A. Cadete

(D).C. Hygino da Cruz Jr. Clínica de Diagnóstico por Imagem Rio de Janeiro, Brazil 

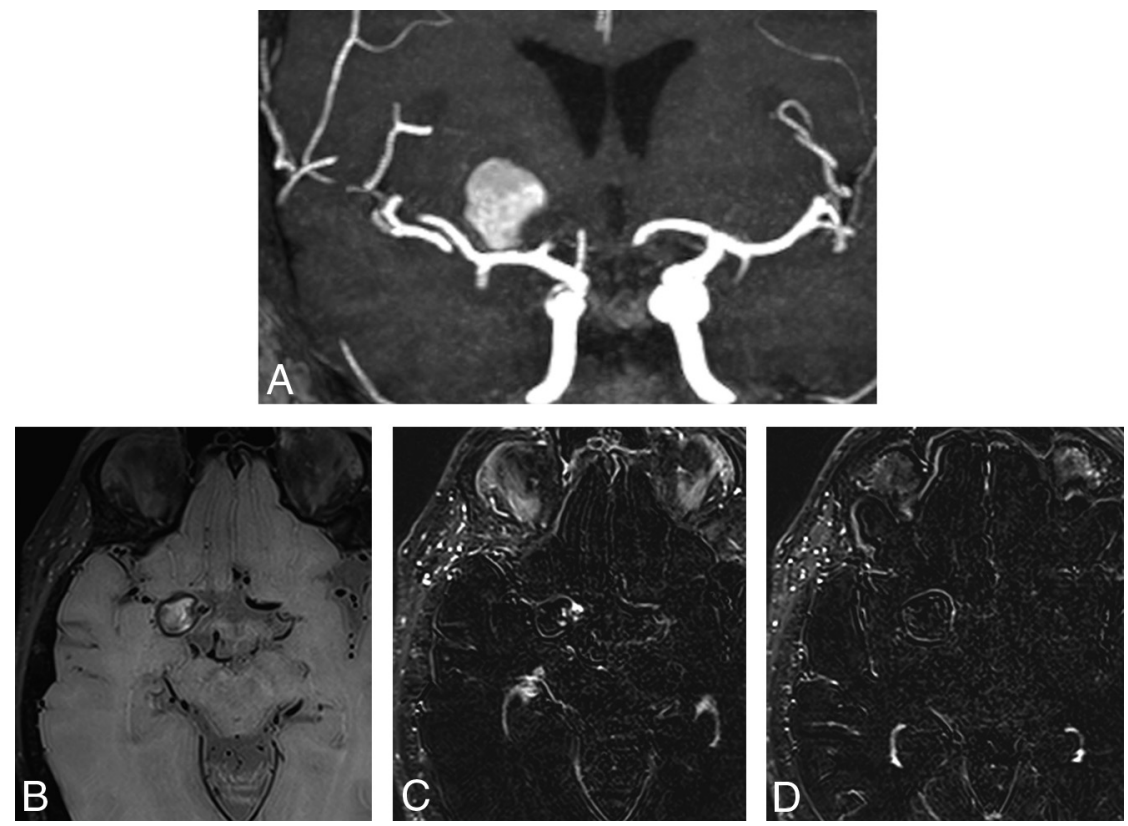

FIGURE. A, Maximum intensity projection of TOF-MR angiography shows an aneurysm adjacent to the Al portion of the right anterior cerebral artery. $B, T 1$ black-blood vessel wall imaging without contrast shows a hyperintense signal area inside the aneurysm due to treatment-induced thrombosis or slow flow. $C$ and $D$, Subtraction of precontrast from postcontrast enhanced vessel wall images shows a thin rim of enhancement around the aneurysm wall and the flow diverter. 